\title{
Revisiting the Slichter mode of Mercury in the context of differentiated models ${ }^{\star}$ (Research Note)
}

\author{
A. Escapa ${ }^{1, \star \star}$ and T. Fukushima ${ }^{2}$ \\ 1 Dept. Mechanical, Informatics, and Aerospace Engineering, University of León, Campus de Vegazana, s/n, 24071 León, Spain \\ e-mail: Alberto.Escapa@ua.es \\ 2 National Astronomical Observatory, 2-21-1 Ohsawa, Mitaka, 181-8588 Tokyo, Japan \\ e-mail: Toshio.Fukushima@nao.ac.jp
}

Received 23 February 2015 / Accepted 12 October 2015

\begin{abstract}
We revisit the Slichter mode of a set of differentiated Mercury models that were considered previously. We apply a different and independent formalism, based on variational principles of mechanics, to analytically model the internal translations of a body with a global internal perfect fluid layer limited by two differentiated rigid solid constituents that have a spherical symmetric mass distribution. The Slichter mode is specified by providing the time evolution of the barycenters of the solid constituents, the amplitude ratio of the mantle to the inner core, and the period of the oscillatory motion. All these parameters only depend on the mass of the body, the mass of the inner core, the density of the fluid, and the mean density of the inner core. For previously developed Mercury models we find, in contrast to other results, that there are no discontinuities in the Slichter period when passing from undifferentiated to differentiated inner cores. Hence, in a general situation, a potential detection of the Slichter mode cannot determine the differentiation of the inner core. We also find that the BepiColombo mission may be able to detect the Slichter mode caused by an impactor meteroid with a mass of about $10^{12} \mathrm{~kg}$ in the most favorable of circumstances. This shows that a measurable excitation of the mode by this mechanism is even more unlikely than was previously established, where a mass about $10^{10} \mathrm{~kg}$ was found.
\end{abstract}

Key words. planets and satellites: individual: Mercury - planets and satellites: interiors - methods: analytical - celestial mechanics

\section{Introduction}

The barycenters of the internal and external solid constituents of a celestial body containing a subsurface global fluid layer can perform relative translations. This motion has an oscillatory nature and is commonly referred to as the Slichter mode, after the research performed by Slichter (1961) for Earth. Observing this mode might provide relevant information with which to constrain some properties of the interior of the body. For this reason, Mercury models have recently also been concerned with the study of this mode in view of the data provided by the MESSENGER spacecraft (e.g., Solomon et al. 2007) or the next ESA/JAXA mission, BepiColombo (e.g., Iess et al. 2009).

The first investigation about a Mercury Slichter mode was reported by Grinfeld \& Wisdom (2005). They considered a body composed of a homogeneous, incompressible, and non-viscous core enclosed by solid homogeneous spherical layers. Some Mercury models, however, can lead to an inner core composed of one layer of iron and another of iron sulfide (e.g., Van Hoolst \& Jacobs 2003) or other more general kinds of stratification (e.g., Hauck et al. 2013, Rivoldini \& Van Hoolst 2013). Hence, a natural step in the development of analytical models of a Mercury Slichter mode was to consider differentiated solid constituents.

\footnotetext{
* Appendices are available in electronic form at http://www. aanda.org

$\star \star$ On leave from department of Applied Mathematics, University of Alicante, PO Box 99, 03080 Alicante, Spain.
}

This task was accomplished by Coyette et al. (2012), who, following the same method as Grinfeld \& Wisdom (2005), described the internal oscillations of Mercury models composed of a crust-mantle layer, a fluid outer core, and a rigid inner core that, when reaching the eutectic composition, differentiates into an sphere covered by a spherical shell consisting of substances of different nature (Van Hoolst \& Jacobs 2003).

The results by Coyette and collaborators suggest a significant numerical difference for the Slichter modes in the differentiated and undifferentiated cases (Coyette et al. 2012, Fig. 4). The authors also discussed the mass of a meteoroid that would be necessary to excite the Slichter mode (Coyette et al. 2012, Fig. 8) and be detected by the BepiColombo altimeter. Both results seem misleading, particularly considering close studies by Escapa (2012) and Coyette \& Van Hoolst (2014) for the Slichter modes of large icy bodies.

The motivation of this article is to throw light on these questions. Hence, we aim at obtaining results by means of an alternative and independent analytical model of the Slichter mode based on the variational approach constructed in Escapa \& Fukushima (2011).

The paper is structured as follows. In Sect. 2 we model the internal translational dynamics of the considered body by means of the variational formalism by Escapa \& Fukushima (2011). The theory, although being general, is applied to differentiated models of Mercury (Van Hoolst \& Jacobs 2003). We analyze the dynamics of some of the models in Sect. 3 and discuss the 
possibility of distinguishing between differentiated or undifferentiated models based on the features of their Slichter modes.

In Sect. 4 we conclude this investigation and compare the obtained results with those reported by Coyette et al. (2012), explaining the probable sources of the discrepancies with respect to the Slichter period and to the minimum mass of a meteoroid needed to excite the Slichter mode. The paper is completed with two appendices.

\section{Dynamics of the system}

\subsection{Model}

We consider a body composed of a rigid spherical shell that encloses a homogeneous, incompressible, and non-viscous fluid containing a spherical rigid body. We refer to them as mantle (subscript $m$ ), fluid core (subscript $f$ ), and inner core (subscript $s$ ), respectively. For instance, the barycenter position vectors are denoted by $\boldsymbol{R}_{m}, \boldsymbol{R}_{f}$, and $\boldsymbol{R}_{s}$.

The solid layers have a mass density function with spherical symmetry with respect to the barycenter of each layer. This structure is more general than considering them to be formed by a finite number of homogeneous spherical layers (Coyette et al. 2012; Coyette \& Van Hoolst 2014).

Although the characteristics of the rigid layers are different, the dynamics can be described following the variational method employed by Escapa \& Fukushima (2011). Hence, we only briefly describe the main steps of the development.

The internal translational oscillations are modeled through

$\boldsymbol{r}=\boldsymbol{R}_{s}-\boldsymbol{R}_{m}, \alpha=-\frac{m_{s}}{m}\left(1-\frac{\rho_{f}}{\bar{\rho}_{s}}\right)$,

which allows us to write (Escapa \& Fukushima 2011)

$\boldsymbol{R}_{m}=\alpha \boldsymbol{r}, \boldsymbol{R}_{f}=-\frac{\alpha\left(m_{m}+m_{s}\right)+m_{s}}{m_{f}} \boldsymbol{r}, \boldsymbol{R}_{s}=(1+\alpha) \boldsymbol{r}$,

$m_{i}$, with $i=m, f, s$, being the mass of each layer and $m$ the total mass of the body. These formulas are inherited for the time derivatives of these vectors, the velocities $\boldsymbol{V}_{m}, \boldsymbol{V}_{f}, \boldsymbol{V}_{s}$, and $\boldsymbol{v}$.

These equations are similar to Eqs. (9) in Escapa \& Fukushima (2011), substituting the former density of the inner core by its mean density $\bar{\rho}_{s}$ in the definition of $\alpha$ (Eq. (1)).

\subsection{Equations of motion and their solution}

The dynamics of the system stems from the Lagrange equations

$\frac{\mathrm{d}}{\mathrm{d} t}\left(\frac{\partial \mathcal{L}}{\partial \boldsymbol{v}}\right)-\frac{\partial \mathcal{L}}{\partial \boldsymbol{r}}=0$

joined to the initial conditions $\boldsymbol{r}\left(t_{0}\right)=\boldsymbol{r}_{0}$ and $\boldsymbol{v}\left(t_{0}\right)=\boldsymbol{v}_{0}$. Here, $\mathcal{L}$ is the Lagrangian of the system that is the difference between the kinetic $\mathcal{T}$ and the potential energies $\mathcal{V}$.

The kinetic energy (Escapa \& Fukushima 2011) is

$\mathcal{T}=\frac{1}{2}\left[m_{m} \alpha^{2}+m_{f} \gamma+m_{s}(1+\alpha)^{2}\right] \boldsymbol{v}^{2}$,

where $\gamma$ depends on $\alpha$ and other geometrical parameters of the model (Escapa \& Fukushima 2011, Eq. (36)).

The computation of the gravitational potential energy is the main difference with respect to the homogeneous solid constituents case. It can be accomplished by following similar guidelines to those developed in Escapa \& Fukushima (2011), which are shown schematically in Appendix A. This leads to

$\mathcal{V}=\frac{2 \pi}{3} G \rho_{f} m_{s}\left(1-\frac{\rho_{f}}{\bar{\rho}_{s}}\right) \boldsymbol{r}^{2}$,

where $G$ is the universal constant of gravitation ${ }^{1}$.

Equations (4) and (5) allow us to write

$\mathcal{L}=\mathcal{T}-\mathcal{V}=\frac{1}{2} m^{*} \boldsymbol{v}^{2}-\frac{1}{2} k^{*} \boldsymbol{r}^{2}$,

with

$m^{*}=m_{m} \alpha^{2}+m_{f} \gamma+m_{s}(1+\alpha)^{2}, k^{*}=\frac{4 \pi}{3} G \rho_{f} m_{s}\left(1-\frac{\rho_{f}}{\bar{\rho}_{s}}\right)$

This is the Lagrangian of an isotropic, or space, harmonic oscillator of frequency $\omega=\sqrt{k^{*} / m^{*}}$. Therefore, the evolution of $\boldsymbol{r}$ is

$\boldsymbol{r}(t)=\boldsymbol{r}_{0} \cos (\omega t)+\frac{\boldsymbol{v}_{0}}{\omega} \sin (\omega t)$

where $\boldsymbol{r}_{0}$ and $\boldsymbol{v}_{0}$ provide the initial conditions of the motion. This expression and Eq. (2) entail that the barycenters of the mantle and the inner core oscillate with the same frequency $\omega$.

The ratio between the moduli of their barycenters is ${ }^{2}$

$\eta=\frac{\left|\boldsymbol{R}_{m}(t)\right|}{\left|\boldsymbol{R}_{s}(t)\right|}=\frac{|\alpha|}{|1+\alpha|}=\left(1-\frac{\rho_{f}}{\bar{\rho}_{s}}\right)\left[\left(\frac{m}{m_{s}}-1\right)+\frac{\rho_{f}}{\bar{\rho}_{s}}\right]^{-1}$.

This quantity is independent of time, therefore it also represents the ratio between the velocity moduli of the barycenters.

The period of the oscillation, $T=2 \pi / \omega$, can be cast (Escapa \& Fukushima 2011) as

$T=\sqrt{\frac{3 \pi}{G} \frac{1+\frac{1}{2} \frac{\rho_{f}}{\bar{\rho}_{s}}+\frac{3}{2}\left(\frac{\rho_{f}}{\bar{\rho}_{s}}\right)^{2} \frac{m_{s}}{m_{f}}-\frac{m_{s}}{m}\left(1-\frac{\rho_{f}}{\bar{\rho}_{s}}\right)^{2}}{\rho_{f}\left(1-\frac{\rho_{f}}{\bar{\rho}_{s}}\right)}}$.

The mantle only influences this expression in an indirect way through $m$. The inner core appears through its mass $m_{s}$, but also through its mean density. This means that if the period of the oscillation were detected, we could verify the existence of an inner core, but we could not determine unambiguously whether it has several differentiated layers or not, a result anticipated in Escapa (2012).

However, as recognized in Earth studies (e.g., Rosat et al. 2006), this potential detection could constrain the mean density jump at the inner core boundary (ICB)

$\Delta \bar{\rho}_{s f}=\bar{\rho}_{s}-\rho_{f}=\bar{\rho}_{s}\left(1-\frac{\rho_{f}}{\bar{\rho}_{s}}\right)$,

since the period is very sensitive to this parameter because of it is the denominator of Eq. (10).

\footnotetext{
1 We take $G=6.674 \times 10^{-11} \mathrm{~m}^{3} \mathrm{~kg}^{-1} \mathrm{~s}^{-2}$ (Mohr et al. 2008).

2 We call $\eta$ the amplitude ratio of the mantle to the inner core.
} 


\section{Internal translational dynamics of differentiated Mercury models}

\subsection{Interior models of Van Hoolst \& Jacobs (2003)}

We used the interior models of Mercury built by Van Hoolst \& Jacobs (2003) and refer to this work for a comprehensive description. They are also the basis for the models developed in Coyette et al. (2012).

The models consist of a crust plus mantle external layer that contains a core composed of a mixture of $\mathrm{Fe}$ with iron sulfide FeS. The initial concentration of sulfur is a parameter of the model $x_{\mathrm{S}}^{\text {in }}$, which can be related to the initial concentration of $\mathrm{FeS} x_{\mathrm{FeS}}^{\text {in }}$. It also provides the initial density of the core, which at this point is entirely fluid

$\rho_{f}^{\text {in }}=\left[\frac{x_{\mathrm{FeS}}^{\text {in }}}{\rho_{\mathrm{FeS}}^{l}}+\frac{1-x_{\mathrm{FeS}}^{\text {in }}}{\rho_{\mathrm{Fe}}^{l}}\right]^{-1}$,

the superscript $l$ denoting liquid substances. Van Hoolst \& Jacobs (2003) computed the density of a liquid substance from its solid counterpart $\rho^{s}$, superscript $s$, by prescribing the relative value of the solid-to-fluid density difference $\Delta \rho^{s l} / \rho^{s}=1-\rho^{l} / \rho^{s}$.

As a result of the cooling of the planet, the model evolves from a completely liquid core to an entirely solid core. This evolution is characterized by the radius of the inner core $d_{s}$, which changes from 0 to $d_{s}^{\text {fin }}$, fulfilling some constraints. For example, it is required to keep the planet mass $m$ and radius $d_{m}$, and other properties that implicitly depend on $x_{\mathrm{S}}^{\text {in }}$ as the mass of the core $m_{c}$

$m_{\mathrm{c}}=\frac{4}{3} \pi d_{m}^{3} \rho_{f}^{\text {in }}\left(\frac{\bar{\rho}-\rho_{m}^{\mathrm{in}}}{\rho_{f}^{\mathrm{in}}-\rho_{m}^{\mathrm{in}}}\right)$,

where $\bar{\rho}$ and $\rho_{m}^{\text {in }}$ denote the known mean density of the planet and the initial density of the mantle.

This evolutionary process is assumed to consist of a precipitation of pure iron from the liquid core, entailing the sulfur enrichment of fluid concentration, which changes the value of its density. When the fluid reaches its eutectic composition, with a sulfur concentration $x_{\mathrm{FeS}}^{\text {eut }}$ and density

$\rho_{f}^{\text {eut }}=\left[\frac{x_{\mathrm{FeS}}^{\mathrm{eut}}}{\rho_{\mathrm{FeS}}^{l}}+\frac{1-x_{\mathrm{FeS}}^{\mathrm{eut}}}{\rho_{\mathrm{Fe}}^{l}}\right]^{-1}$,

the inner core passes from an undifferentiated stage to a differentiated one. This point corresponds to the value

$d_{s}^{\text {eut }}=\left[\frac{3 m_{c}}{4 \pi \rho_{\mathrm{Fe}}^{s}}\left(1-\frac{x_{\mathrm{FeS}}^{\text {in }}}{x_{\mathrm{FeS}}^{\text {eut }}}\right)\right]^{1 / 3}$.

In the undifferentiated stage, the inner core is a solid Fe sphere of radius $d_{s}$; in the differentiated stage, this solid Fe sphere, now with constant radius $d_{s}^{\text {eut }}$, is covered by a solid Fe-FeS (at the eutectic composition) shell with thickness $d_{s}-d_{s}^{\text {eut }}$ and density

$\rho_{s_{e}}=\left(1-\frac{\Delta \rho^{s l}}{\rho^{s}}\right)^{-1} \rho_{f}^{\text {eut }}$.

This evolution ends in a complete solid core with radius

$d_{s}^{\mathrm{fin}}=\sqrt[3]{\frac{\rho_{f}^{\text {in }}}{\rho_{s_{e}}}\left(d_{f}^{\mathrm{in}}\right)^{3}+\left(1-\frac{\rho_{\mathrm{Fe}}^{s}}{\rho_{s_{e}}}\right)\left(d_{s}^{\mathrm{eut}}\right)^{3}}$, where the initial radius of the core $d_{f}^{\text {in }}$ can be computed from the core mass and density (Eqs. (12) and (13)) at this state.

The former expressions allow us to express the mean density function of the inner core as

$\bar{\rho}_{s}\left(d_{s}\right)=\left\{\begin{array}{cc}\rho_{\mathrm{Fe}}^{s}, & 0<d_{s} \leq d_{s}^{\text {eut }}, \\ \rho_{\mathrm{Fe}}^{s}\left(\frac{d_{s}^{\text {eut }}}{d_{s}}\right)^{3}+\rho_{f}^{\text {eut }} \frac{1-\left(\frac{d_{s}^{\text {eut }}}{d_{s}}\right)^{3}}{\left(1-\frac{\Delta \rho^{s l}}{\rho^{s}}\right)}, & d_{s}^{\text {eut }}<d_{s}<d_{s}^{\text {fin }} .\end{array}\right.$

From the mean density, the mass of the inner core is obtained,

$m_{s}\left(d_{s}\right)=\frac{4}{3} \pi d_{s}^{3} \bar{\rho}_{s}\left(d_{s}\right)$.

In a similar way, the density of the fluid turns out to be

$\rho_{f}\left(d_{s}\right)=\left\{\left[\begin{array}{cl}\left.x_{\mathrm{FeS}}^{\mathrm{in}} \frac{\left(\frac{1}{\rho_{\mathrm{FeS}}^{l}}-\frac{1}{\rho_{\mathrm{Fe}}^{l}}\right)}{1-\frac{m_{s}\left(d_{s}\right)}{m_{\mathrm{c}}}}+\frac{1}{\rho_{\mathrm{Fe}}^{l}}\right]^{-1}, \quad 0<d_{s} \leq d_{s}^{\text {eut }}, \\ \rho_{f}^{\text {eut }}, & d_{s}^{\text {eut }}<d_{s}<d_{s}^{\text {fin }} .\end{array}\right.\right.$

Since these interior models maintain the mass of the core invariable (Van Hoolst \& Jacobs 2003), the mass of the fluid is given by

$m_{f}\left(d_{s}\right)=m_{\mathrm{c}}-m_{s}\left(d_{s}\right)$.

We represent the functions $\bar{\rho}_{s}\left(d_{s}\right)$ and $\rho_{f}\left(d_{s}\right)$, and the relative mean density jump $\Delta \bar{\rho}_{s f}\left(d_{s}\right) / \bar{\rho}_{s}\left(d_{s}\right)$ in Fig. 1 . To this end, we used the numerical values provided in Van Hoolst \& Jacobs (2003), with $\bar{\rho}=5433 \mathrm{~kg} \mathrm{~m}^{-3}$ and $\rho_{\mathrm{Fe}}^{l}=7874 \mathrm{~kg} \mathrm{~m}^{-3}$, and considered four different initial concentrations of $x_{\mathrm{S}}^{\mathrm{in}}: 0.1,4.5$, 9 , and $14 \mathrm{wt} \%$. The graphs of $\bar{\rho}_{s}\left(d_{s}\right)$ and $\rho_{f}\left(d_{s}\right)$ coincide $^{3}$ with those of Van Hoolst \& Jacobs (2003, Fig. 5), which can be considered as a verification of Eqs. (18) and (20).

\subsection{Period of the oscillations and amplitude ratio of the mantle to the inner core}

The substitution of the former expressions into the interior models of Mercury in Eqs. (10) and (9) gives the values of the period of the translational oscillations $T$ and the amplitude ratio of the mantle to the inner core $\eta$, which must be written as $T\left(d_{s}\right)$ and $\eta\left(d_{s}\right)$. This determines the Slichter mode of each Mercury model as function of $d_{s}$. The nature of this dependence is better understood by considering both the previous analytical formulas and the plots of Fig. 2.

We represent the functions $T\left(d_{s}\right)$ and $\eta\left(d_{s}\right)$ for the same initial concentrations of $x_{\mathrm{S}}^{\text {in }}$ as in Fig. 1, joining the sharp points of the graphs corresponding to the same initial sulfur concentration with dashed vertical straight lines passing through the eutectic point $\left(d_{s}^{\text {eut }}, 0\right)$. In contrast to Fig. 1 , we have considered a slightly shorter interval of variation of $d_{s}$. By doing so, we excluded the situations where the mass of the solid inner core or of the fluid core is zero, since in these cases there are no internal oscillations.

Figure 2 (top) shows that in the first stages of the evolution the periods last from about three to eight hours, depending on

3 Van Hoolst \& Jacobs (2003, Fig. 6) represented the relative local density jump at ICB, not the relative mean density jump. 


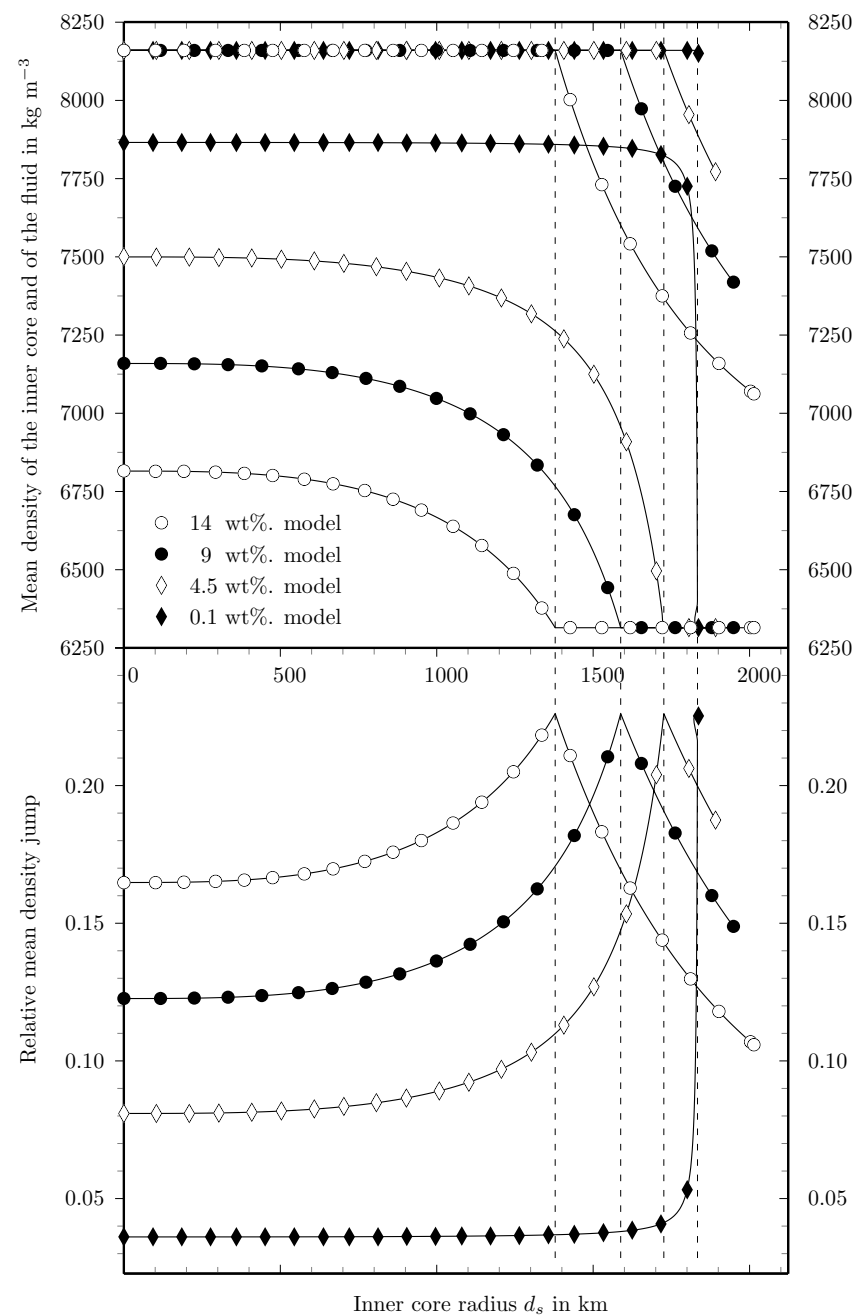

Fig. 1. Mean density of the inner core and density of the fluid (top) and relative mean density jump (bottom) as functions of the inner core radius for some interior models of Mercury. For each model $\rho_{f}\left(d_{s}\right)<\bar{\rho}_{s}\left(d_{s}\right)$, the vertical dashed line passing through its eutectic point $\left(d_{s}^{\text {eut }}, 0\right)$. The symbols are spaced apart for illustrative purposes.

the initial sulfur concentration. Then, they increase as a consequence of the progressive solidification of the core. These features can be understood from the analytical expression of the period (Eq. (10)) and the representation of $\bar{\rho}_{s}\left(d_{s}\right), \rho_{f}\left(d_{s}\right)$, and $\Delta \bar{\rho}_{s f}\left(d_{s}\right) / \bar{\rho}_{s}\left(d_{s}\right)$ provided in Fig. 1 . The models with a poorer initial sulfur concentration lead to higher values of the period, as a consequence of the dependence of $T\left(d_{s}\right)$ on the relative mean density jump at ICB (Eqs. (10) and (11), and Fig. 1, bottom).

We emphasize that the period does not experience any kind of jump when surpassing the eutectic point. This is a consequence of the continuity of $T\left(d_{s}\right)$ as a function of $d_{s}$, derived through Eq. (9) and that of the mass density functions. In particular, we note that the relative mean density jump at ICB (Fig. 1, bottom) is continuous everywhere.

The involved orders of magnitude become clearer through the results in Table 1 . There, we computed the period corresponding to each model for three different values of $d_{s}$. These common values were selected to represent an initial, $d_{s}=$ $100 \mathrm{~km}$, medium, $d_{s}=1600 \mathrm{~km}$, and final stage, $d_{s}=1835 \mathrm{~km}$ in the evolution of the planet. They also provide different scenarios in the process of differentiation of the models: all of them are undifferentiated for $d_{s}=100 \mathrm{~km}$ and differentiated for $d_{s}=1835 \mathrm{~km}$. For $d_{s}=1600 \mathrm{~km}$ the models with lower

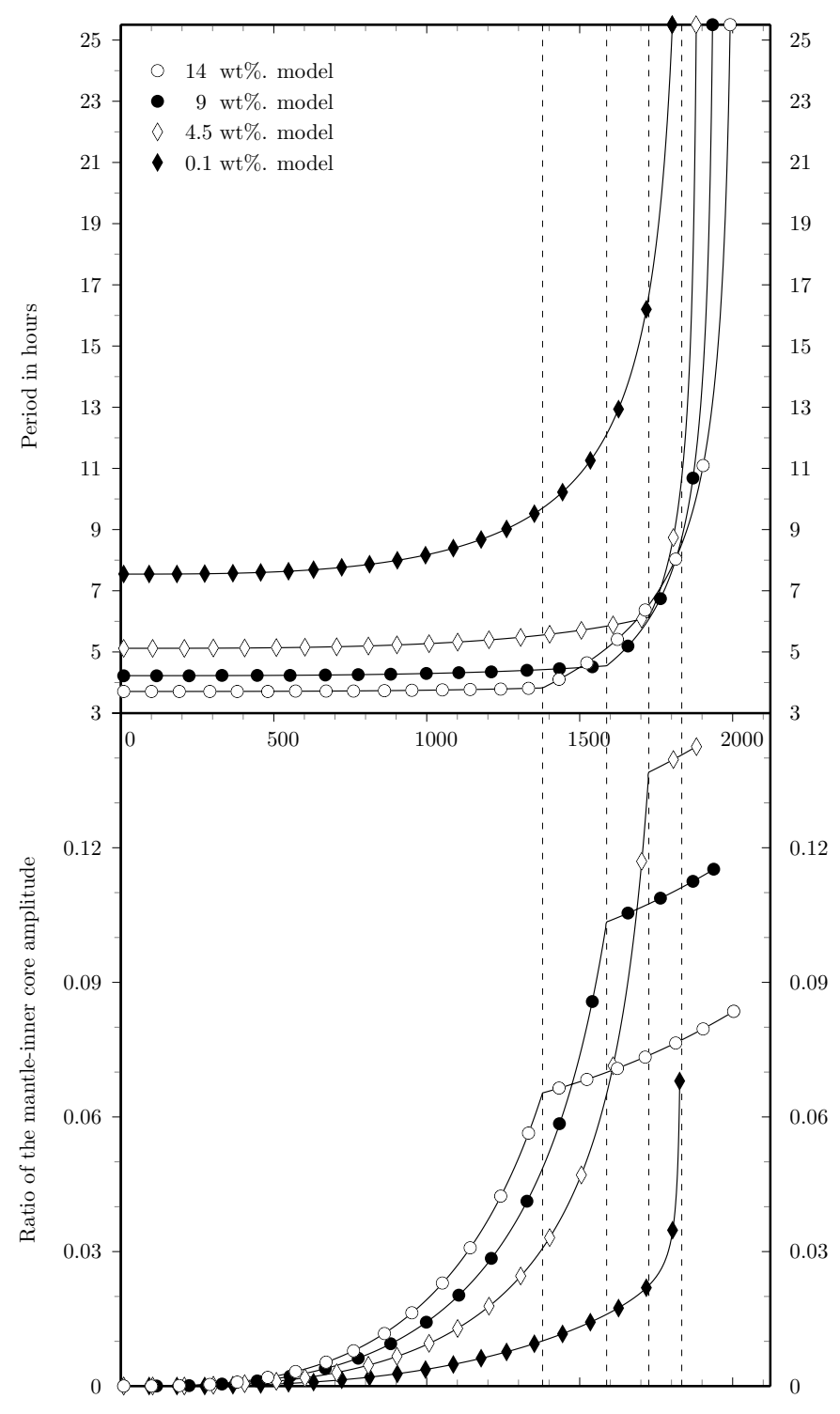

Inner core radius $d_{s}$ in $\mathrm{km}$

Fig. 2. Slichter period (top) and amplitude ratio of the mantle to the inner core (bottom) as functions of the inner core radius for the same interior models of Mercury as were considered in Fig. 1.

Table 1. Period $T$ (in hours) of the internal translational oscillations for different Mercury models and values of the inner core radius.

\begin{tabular}{cccc}
\hline \hline Model & \multicolumn{3}{c}{$d_{s}(\mathrm{~km})$} \\
\hline$x_{S}^{\text {in }} \%$ & 100 & 1600 & 1835 \\
\hline 0.01 & 7.55 & 12.35 & 51.47 \\
4.5 & 5.12 & 5.87 & 10.84 \\
9 & 4.23 & 4.65 & 8.79 \\
14 & 3.71 & 5.22 & 8.56 \\
\hline
\end{tabular}

concentrations are not differentiated, whereas the two remaining concentrations are because they have exceeded their eutectic points (Eq. (15)).

With respect to the graph of the amplitude ratio of the mantle to the inner core, Fig. 2 (bottom) shows that all the considered initial sulfur concentrations models lead to a relatively low value of $\eta\left(d_{s}\right)$. This means that the displacement of the mantle barycenter is, at least, about five times smaller than that of 
the inner core. This fact can be elucidated by writing Eq. (9) in the form

$\eta\left(d_{s}\right)=\left(\frac{m}{m_{s}\left(d_{s}\right)} \frac{\bar{\rho}_{s}\left(d_{s}\right)}{\Delta \bar{\rho}_{s f}\left(d_{s}\right)}-1\right)^{-1}$.

For all the interior models considered, the lowest value of $m / m_{s}\left(d_{s}\right)$ is about 1.5 , which roughly represents the $66 \%$ of the mass of Mercury. However, the key point providing the order of magnitude of $\eta\left(d_{s}\right)$ is the low value of the relative mean density jump, which never exceeds 0.25 (Fig. 1, bottom) because of the close rheological properties of the fluid and the solid inner core of the Mercury models ${ }^{4}$.

\subsection{Observation of the period and undifferentiated and differentiated models}

The former results also allows elucidating to what extent the internal dynamics is sensitive to a possible differentiation of the inner core. Figure 2 shows a change in the trend of $T\left(d_{s}\right)$ and $\eta\left(d_{s}\right)$ when reaching the eutectic point, which determines the appearance of a differentiated inner core. We might wonder whether a potential observation of the free period of the internal oscillations would allow distinguishing between differentiated or undifferentiated models.

For the considered four interior models of Mercury, and assuming that the radius of the inner core is known, some values of the period would lead unequivocally to a particular interior model of Mercury. In contrast, other values would not lead to a unique interior model because some graphs of their periods intersect (Fig. 1, top). In other words, since the period is mainly sensitive to the mean density jump, the same numerical value can be associated with differentiated or undifferentiated inner cores with different initial sulfur concentrations. For example, in Table 1 we see that a period value of about five hours, with $d_{s}=1600 \mathrm{~km}$, could correspond to models with an undetermined value of $x_{\mathrm{S}}^{\text {in }}$ roughly running from 4.5 to $14 \mathrm{wt} \%$, some of which are differentiated and others undifferentiated.

Hence, in a general situation these potential observations by themselves cannot determine unambiguously whether the inner core is undifferentiated or differentiated in two or more sublayers. However, the period value can constrain the value of the mean density jump $\Delta \bar{\rho}_{s f}\left(d_{s}\right)$, which in combination with other methods and models can throw light onto the interior structure of the body.

\section{Discussion}

\subsection{Slichter period features}

We compared our results with those presented in Coyette et al. (2012) concerning the Slichter period, providing at the same time its main characteristics.

The structure of the body considered to develop a model of the Slichter mode is similar in both works. The main difference lies in our generalization to the case in which the solid layers have an arbitrarily spherically symmetric mass distribution. In contrast, Coyette et al. (2012) considered that the solid layers are differentiated into a finite number of homogeneous sublayers.

Another essential difference is the framework in which the internal translational motion is modeled. Here,

\footnotetext{
4 The relative density contrast of some icy moons can reach values about 0.7 (Escapa \& Fukushima 2011).
}

we have followed the variational approach developed in Escapa \& Fukushima (2011), whereas Coyette et al. (2012) basically employed the method reported by Grinfeld \& Wisdom (2005).

Although the variational formalism provides a neat analytical description of the dynamics, both approaches are physically valid and must return equivalent solutions. We should therefore recover the results of Coyette et al. (2012) when particularizing to the differentiation model of the solid layers considered in that work.

This can be accomplished by reducing Eqs. (10) and (9), which provide the characteristics of the Slichter mode, to the kind of body described in Coyette et al. (2012). To do this, we have to express the mass of the mantle, the fluid, and the inner core and its mean density in terms of the densities and radius of the finite homogeneous sublayers considered by these authors.

This would enable us to analytically compare our results with those derived in Coyette et al. (2012). However, this comparison can only be made partially because Coyette and collaborators only provided a closed expression for the amplitude ratio of the mantle to the inner core ${ }^{5}$ (Coyette et al. 2012, Eq. (23)). This equation is equivalent to the corresponding one derived in this work (Eq. (9)), as we show in Appendix B.

For the period of the internal oscillations no explicit analytical formulas were provided. Nevertheless, Coyette et al. (2012) derived different graphs that show the characteristics of the period for a set of particular interior models of Mercury. We can make a qualitative comparison by comparing them with the plot displayed in this investigation (Fig. 2).

With this aim, it is first necessary to estimate the influence of the used particular models on the results shown in these graphs. As we mentioned in Sect. 3.1, the interior models developed in Coyette et al. (2012) follow the same guidelines as those of Van Hoolst \& Jacobs (2003), which we have considered in this investigation, but some modifications were introduced. These are related not to their global structure, but mainly to new experimental data about the properties of $\mathrm{Fe}$ and $\mathrm{FeS}$ at the internal conditions of Mercury (Coyette et al. 2012), providing different numerical values than for some of the parameters of Van Hoolst \& Jacobs (2003).

We can obtain an idea of the influence of these changes when we compare the graphs of the amplitude ratio of the mantle to the inner core presented ${ }^{6}$ in Coyette et al. (2012, Fig. 3) and here (Fig. 2, bottom), since we have proved previously that these expressions are algebraically identical. This comparison shows that the two representations are qualitatively equivalent, the amplitude ratio of the mantle to the inner core exhibiting an identical behavior as a function of radius of the inner core for the different considered initial sulfur concentrations ${ }^{7}$. From a qualitative point of view, the differences in the numerical values employed in both studies are therefore not significant and do not change the general pictures of the Slichter mode features.

Next, we performed a similar analysis and compared the graphs of the internal oscillation periods displayed in Coyette et al. (2012, Fig. 4) and here (Fig. 2, top). They are qualitatively

\footnotetext{
5 The amplitude of the normal velocity of the mantle and the inner core (Coyette et al. 2012) is equal to the amplitude of the mantle to the inner core $\eta$.

6 Coyette et al. (2012) reported the amplitude ratio of the mantle to the inner core in percent. The label of the ordinate axis of that figure should appear as "Amplitude ratio mantle/inner core (\%)".

7 The quantitative differences are significantly reduced if we take in our computations the eutectic concentration equal to $16 \%$ (Coyette et al. 2012) instead of the value $22.2 \%$ (Van Hoolst \& Jacobs 2003).
} 
very different, since the plots by Coyette et al. (2012) show a discontinuity in the period at the eutectic point, that is, in the beginning of the differentiated scenario. As the authors correctly recognized (Coyette et al. 2012), this jump would entail a divergent behavior of the undifferentiated and differentiated Mercury models (Coyette et al. 2012, Fig. 5). In contrast, our representation is continuous everywhere and implies no basic difference in the Slichter mode of undifferentiated and differentiated Mercury models, as we discussed in Sect. 3.

Hence, there must be some kind of deficiency in the treatment given by Coyette et al. (2012), which might also be present in the subsequent figures of their study. The origin of this deficiency can very likely be attributed to some error in the numerical code used to calculate the Slichter mode period, as was reported by the authors (Coyette 2012, 2014, priv. comm.). This explanation is corroborated by a result provided recently in Coyette \& Van Hoolst (2014, Eqs. (13)-(15)).

Summing up, there are no abrupt changes in the Slichter period for differentiated and undifferentiated models (Fig. 2). This means that in a general situation a potential observation of the Slichter period would not allow distinguishing between the two scenarios.

\subsection{Slichter mode excitation by a meteoroid impact}

It may be not out of place to discuss the possibility of a meteoroid exciting the Slichter mode. If that excitation had enough energy, the motion might be detected from an onboard altimeter carried by some space mission such as MESSENGER or BepiColombo (Coyette et al. 2012).

We followed the same hypothesis as in Coyette et al. (2012). They provided the most favorable situation that would enable such a detection. It was assumed that the energy efficiency of the impact $k$ has the expression ${ }^{8}$ given by Shishkin (2007, Eq. (3.4)), and that all this energy is absorbed by the Slichter mode. For this we have

$2 \mathcal{E}_{\mathrm{SM}}=k\left(m_{\mathrm{I}} \boldsymbol{V}_{\mathrm{I}}^{2}\right)=\tilde{k} m_{\mathrm{I}}^{-2 / 9}\left(m_{\mathrm{I}} \boldsymbol{V}_{\mathrm{I}}^{2}\right)$

where $\mathcal{E}_{\mathrm{SM}}$ is the Slichter mode energy and $\boldsymbol{V}_{\mathrm{I}}$ and $m_{\mathrm{I}}$ are the velocity and mass of the impactor. Following Eqs. (4), (5), and (7), $\mathcal{E}_{\mathrm{SM}}$ is

$2 \mathcal{E}_{\mathrm{SM}}=2 \mathcal{T}+2 \mathcal{V}=m^{*} \boldsymbol{v}^{2}+k^{*} \boldsymbol{r}^{2}$

Since the system is conservative, we can obtain its value from the state with zero velocity (e.g., Coyette \& Van Hoolst 2014, Eq. (30)) or from the state with the highest velocity $(\boldsymbol{r}=\mathbf{0})$

$2 \mathcal{E}_{\mathrm{SM}}=m^{*} \boldsymbol{v}_{\max }^{2}=\left[m_{m} \alpha^{2}+m_{f} \gamma+m_{s}(1+\alpha)^{2}\right] \boldsymbol{v}_{\max }^{2}$.

We note that the contribution from the kinetic energy of the fluid, $\gamma$ term, is absent in Coyette et al (2012, Eq. (56)). However, the Slichter mode necessarily implies the motion of the fluid when the velocity of the rigid constituents is not nil (Eq. (2)).

Equation (8) entails that $\boldsymbol{v}_{\max }^{2}=\omega^{2} \boldsymbol{r}_{\max }^{2}$, which in combination with the definition of $\omega$ and Eqs. (2) and (9) leads to

$2 \mathcal{E}_{\mathrm{SM}}=\frac{m^{*} \omega^{2}}{\alpha^{2}} \boldsymbol{R}_{m \max }^{2}=\frac{k^{*}}{\alpha^{2}} \boldsymbol{R}_{m \max }^{2}=k^{*}\left(1+\frac{1}{\eta}\right)^{2} \boldsymbol{R}_{m \max }^{2}$.

8 This expression is only valid for an aerolite impact (Shishkin 2007).

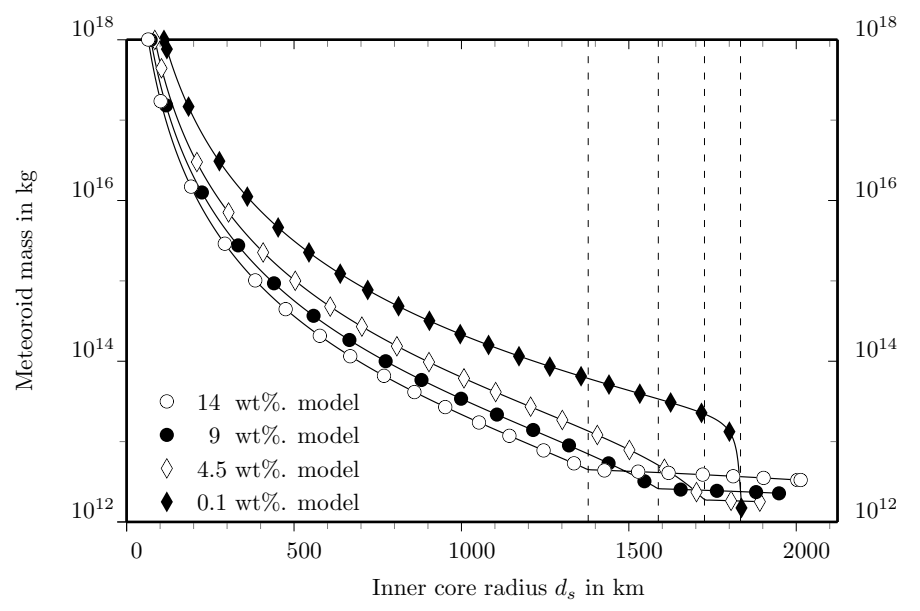

Fig. 3. Minimum mass of a meteoroid necessary to produce a Slichter mode observable by the BepiColombo altimeter in the most favorable situation. The interior models of Mercury are the same as in Fig. 1.

This relationship provides the minimum mass of a meteoroid that is necessary to produce a prescribed mantle amplitude displacement of $\boldsymbol{R}_{m \max }^{2}$ value. Indeed, by combining Eqs. (23) and (26), we obtain

$m_{\mathrm{I}}=\left[k^{*}\left(1+\frac{1}{\eta}\right)^{2} \frac{\boldsymbol{R}_{m \max }^{2}}{\tilde{k} \boldsymbol{V}_{\mathrm{I}}^{2}}\right]^{9 / 7}$.

The application of this formula to the Mercury models by Van Hoolst \& Jacobs (2003) considered in Sect. 3.1 determines the minimum mass of the meteoroid as a function of $d_{s}, m_{\mathrm{I}}\left(d_{s}\right)$. From Coyette et al. (2012) we derive the values $V_{\mathrm{I}}=30 \mathrm{~km} \mathrm{~s}^{-1}$ and $\tilde{k}=1.529 \mathrm{~kg}^{2 / 9}$. Assuming also that the BepiColombo laser altimeter BELA provides precise measurements to $1 \mathrm{~m}$ (Iess et al. 2009), that is, $\boldsymbol{R}_{m \text { max }}^{2}=1 \mathrm{~m}$, we can represent $m_{\mathrm{I}}\left(d_{s}\right)$ as a function of $d_{s}$ (Fig. 3).

Figure 3 shows that the minimum mass of the meteoroid that would induce an observable Slichter mode reaches from $10^{18} \mathrm{~kg}$ in the first stages of the evolution to $10^{12} \mathrm{~kg}$ for the largest inner cores. If we compare this graph with that displayed ${ }^{9}$ in Coyette et al. (2012, Fig. 8), two discrepancies are evident.

The first difference is that there are discontinuities in the values of $m_{\mathrm{I}}\left(d_{s}\right)$ when the eutectic points are reached. This difference has a similar cause as explained before for the Slichter period.

The second difference is the value of the minimum mass of the meteoroid for large inner cores. Coyette et al. (2012) reported this value to be about $10^{10} \mathrm{~kg}$ in contrast to the $10^{12} \mathrm{~kg}$ derived here. This difference of two orders of magnitude is due to the absence of the fluid contribution in the energy of the Slichter mode (Coyette et al. (2012, Eq. (56)).

This contribution increases the energy of the Slichter mode in a significant way and must not be neglected. With an impactor density equal to $1000 \mathrm{~kg} \mathrm{~m}^{-3}$ (Coyette et al. 2012), it entails an spherical meteoroid of radius about $600 \mathrm{~m}$. This means an average interval between two impacts on the order of $350 \mathrm{My}$ (Marchi et al. 2005, Eq. (2)), in contrast to the value of $140 \mathrm{My}$ provided by Coyette et al. (2012). Therefore, a potential observation of the Slichter mode if excited by this mechanism is even more unlikely than that concluded in Coyette et al. (2012).

9 The symbol $\eta$ appearing in Eqs. (57) and (59) in Coyette et al. (2012) should appear as $\eta^{-1}$ or be defined as the inverse of their Eqs. (23) and (52). 
A. Escapa and T. Fukushima: Revisiting the Slichter mode of Mercury in the context of differentiated models $(R N)$

Acknowledgements. The authors thank the referees for their suggestions that improved the manuscript. A.E. wishes to express his gratitude to the generous hospitality of the staff of the National Astronomical Observatory of Japan (NAOJ) during his visits made to carry out this work. This investigation has been partially supported by the Generalitat Valenciana project GV/2014/072 and the Spanish government MINECO project AYA2010-22039-C02-02.

\section{References}

Coyette, A., \& Van Hoolst, T. 2014, Icarus, 231, 287

Coyette, A., Van Hoolst, T., \& Dehant, V. 2012, A\&A, 543, A40

Escapa, A. 2012, EPSC 2012 abstract, 209

Escapa, A., \& Fukushima, T. 2011, AJ, 141, 77

Grinfeld, P., \& Wisdom, J. 2005, Phys. Earth Planet. Interiors, 151, 77
Hauck II, S. A., Margot, J. L., Solomon, S. C., et al. 2013, J. Geophys. Res., 118, 1204

Iess, L., Asmarb, S., \& Tortora, P. 2009, Acta Astronaut., 65, 666

MacMillan, W. D. 1958, The theory of the Potential (New York: Dover Publications)

Marchi, S., Morbidelli, A., \& Cremonese, G. 2005, A\&A, 431, 1123

Mohr, P. J., Taylor, B. N., \& Newell, D. B. 2008, Rev. Mod. Phys., 80, 633

Rivoldini, A., \& Van Hoolst, T. 2013, Earth Planet. Sci. Lett., 377, 62

Rosat, S., Rogister, Y., Crossley, D., \& Hinderer, J. 2006, J. Geodyn., 41, 296

Shishkin, N. I. 2007, J. Appl. Mech. Tech. Phys., 48, 145

Slichter, L. 1961, Proc. Natl. Acad. Sci. USA, 47, 186

Solomon, S. C., McNutt, Jr., R. L., Gold, R. E., \& Domingue, D. L. 2007, Space Sci. Rev., 131, 3

Van Hoolst, T. \& Jacobs, C. 2003, J. Geophys. Res., 108, 5121 


\section{Appendix A: Computing the gravitational potential energy of the system}

The gravitational potential energy of $n$ different subsystems with volumes $D_{i}$ and densities $\rho_{i}(\boldsymbol{R})$ (MacMillan 1958) is

$\mathcal{V}=\frac{1}{2} \sum_{i=1}^{n} \sum_{j=1}^{n} \int_{D_{j}} \mathcal{V}_{i}(\boldsymbol{R}) \rho_{j}(\boldsymbol{R}) \mathrm{d} \tau^{3}=\frac{1}{2} \sum_{i=1}^{n} \sum_{j=1}^{n} \mathcal{V}_{i j}$

where $\mathcal{V}_{i j}=\mathcal{V}_{j i}$ and the potential ${ }^{10}$ of each subsystem (MacMillan 1958) is given by

$\mathcal{V}_{i}(\boldsymbol{R})=-G \int_{D_{i}} \frac{1}{\left|\boldsymbol{R}-\boldsymbol{R}^{*}\right|} \rho_{i}\left(\boldsymbol{R}^{*}\right) \mathrm{d} \tau^{* 3}$.

In our case we have three constituents $(i, j=m, f, s)$, hence

$\mathcal{V}=\frac{1}{2} \mathcal{V}_{m m}+\frac{1}{2} \mathcal{V}_{f f}+\frac{1}{2} \mathcal{V}_{s s}+\mathcal{V}_{m f}+\mathcal{V}_{m s}+\mathcal{V}_{f s}$

The difficulty in evaluating this expression is related to the geometry of the region occupied by the fluid. To overcome this problem, we take into account (Escapa \& Fukushima 2011) that

$D_{f}=\left(D_{f}^{(0)} \cup D_{s}^{(0)}\right)-D_{s}$,

the superscript (0) referring to the configuration when $\boldsymbol{R}_{m}=\boldsymbol{R}_{s}$. In this way, from Eqs. (A.2) and (A.4) the gravitational potential of the fluid can be written in the form

$\mathcal{V}_{f}(\boldsymbol{R})=\mathcal{V}_{f a}(\boldsymbol{R})-\mathcal{V}_{f b}(\boldsymbol{R})$.

The potential $\mathcal{V}_{f a}(\boldsymbol{R})$ is that of a fluid with density $\rho_{f}$ and volume given by an sphere of radius $d_{m}$ centered at the mantle barycenter; the potential $\mathcal{V}_{f b}(\boldsymbol{R})$ corresponds to a fluid with density $\rho_{f}$ and volume given by an sphere of radius $d_{s}$ centered at the inner core barycenter.

Accordingly, the gravitational potential energy of the system is

$$
\begin{aligned}
\mathcal{V}= & \frac{1}{2} \mathcal{V}_{m m}+\frac{1}{2}\left(\mathcal{V}_{f_{a} f_{a}}-\mathcal{V}_{f_{b} f_{b}}-2 \mathcal{V}_{f_{a} f_{b}}\right) \\
& +\frac{1}{2} \mathcal{V}_{s s}+\mathcal{V}_{m f}+\mathcal{V}_{m s}+\left(\mathcal{V}_{f_{a} s}+\mathcal{V}_{f_{b} s}\right)
\end{aligned}
$$

The subsystems involved in this equation are spheres or spherical shells with constant or spherical symmetric density functions. Their potentials can be obtained from that of a spherical shell $(B)$ with interior radius $d_{1}$, which would be 0 for a sphere, and exterior radius $d_{2}$, whose density is a piecewise continuous function $\rho$ of the distance from the center. This has the expression $\mathcal{V}_{B}(R)= \begin{cases}-4 \pi G \int_{d_{1}}^{d_{2}} \alpha \rho(\alpha) \mathrm{d} \alpha, & 0 \leq R \leq d_{1}, \\ -G\left(\frac{m(R)}{R}+4 \pi \int_{R}^{d_{2}} \alpha \rho(\alpha) \mathrm{d} \alpha\right), & d_{1} \leq R \leq d_{2}, \\ -G \frac{m}{R}, & d_{2} \leq R .\end{cases}$

If in this equation the density is $\rho(\alpha)=\rho=1$, we recover the formulas given in Escapa \& Fukushima (2011, Eq. (B3) ${ }^{11}$ ).

The application of Eqs. (A.7) to (A.6) provides the constant $k_{1}$, arising from the terms $\mathcal{V}_{m f}$ and $\mathcal{V}_{m s}$, and from $\mathcal{V}_{m m}$, $\mathcal{V}_{f_{a} f_{a}}, \mathcal{V}_{f_{b} f_{b}}, \mathcal{V}_{s s}$, and $\mathcal{V}_{f_{b} s}$. The first group is constant because it

\footnotetext{
${ }^{10}$ Escapa \& Fukushima (2011) denoted by $\mathcal{V}_{D_{j}}(\boldsymbol{R})$ the gravitational potential of a body occupying the volume $D_{j}$ with a mass density equal to 1 .

${ }^{11}$ In the second line of this equation the parentheses $\left(3 d_{2}^{3}-R^{2}\right)$ must appear as $\left(3 d_{2}^{2}-R^{2}\right)$.
}

is the case for the potential $\mathcal{V}_{m}$ when evaluated at interior points of the spherical shell. The second group is constant because it provides the gravitational self-energy of spheres or spherical shells referring to the same region of space.

The remaining terms in $\mathcal{V}$ can be cast as

$\mathcal{V}_{f_{a} s}-\mathcal{V}_{f_{a} f_{b}}=\int_{D_{s}} \mathcal{V}_{f_{a}}(\boldsymbol{R})\left[\rho_{s}\left(\left|\boldsymbol{R}-\boldsymbol{R}_{s}\right|\right)-\rho_{f}\right] \mathrm{d} \tau^{3}$.

With the expression of $\mathcal{V}_{f_{a}}(\boldsymbol{R})$ arising from Eq. (A.7), it reads

$$
\begin{aligned}
\mathcal{V}_{f_{a} s}-\mathcal{V}_{f_{a} f_{b}}= & \int_{D_{s}}\left\{-\frac{2 \pi}{3} G \rho_{f}\left[3 d_{f}^{2}-\left(\boldsymbol{R}-\boldsymbol{R}_{m}\right)^{2}\right]\right\} \\
& \times\left[\rho_{s}\left(\left|\boldsymbol{R}-\boldsymbol{R}_{s}\right|\right)-\rho_{f}\right] \mathrm{d} \tau^{3} .
\end{aligned}
$$

A change of variable $\boldsymbol{R}^{\prime \prime}=\boldsymbol{R}-\boldsymbol{R}_{s}=\boldsymbol{R}-\boldsymbol{R}_{m}-\boldsymbol{r}$ allows computing this integral with respect to a reference system whose origin is located at the center of sphere $D_{s}^{\prime \prime}$. In this way, we obtain

$\mathcal{V}_{f_{a} s}-\mathcal{V}_{f_{a} f_{b}}=\frac{2 \pi}{3} G \rho_{f}\left(m_{s}-\rho_{f} \operatorname{Vol} D_{s}^{\prime \prime}\right) \boldsymbol{r}^{2}+k_{2}$,

where $k_{2}$ comprises all the constant terms resulting from the integration, and Vol $D_{s}^{\prime \prime}=\mathrm{Vol} D_{s}$ is the volume of the inner core.

Therefore, the gravitational potential energy of the system is

$\mathcal{V}=\frac{2 \pi}{3} G \rho_{f} m_{s}\left(1-\frac{\rho_{f}}{\bar{\rho}_{s}}\right) \boldsymbol{r}^{2}+k_{1}+k_{2}$;

since the constant terms play no role for our purposes, we will omit them.

\section{Appendix B: Expression of the amplitude ratio \\ of the mantle to the inner core for the stratification of the rigid layers assumed in Coyette et al. (2012)}

Here, we follow the same notation as Coyette et al. (2012). With this, we can write ${ }^{12}$ for the mass of the mantle, the fluid, and the inner core

$$
\begin{aligned}
& m_{m}=\frac{4 \pi}{3}\left[\rho_{i c+2}\left(R_{i c+2}^{3}-R_{i c+1}^{3}\right)+\ldots+\rho_{N}\left(R_{N}^{3}-R_{N-1}^{3}\right)\right], \\
& m_{f}=\frac{4 \pi}{3} \rho_{i c+1}\left(R_{i c+1}^{3}-R_{i c}^{3}\right), \\
& m_{s}=\frac{4 \pi}{3}\left[\rho_{1} R_{1}^{3}+\rho_{2}\left(R_{2}^{3}-R_{1}^{3}\right)+\ldots+\rho_{i c}\left(R_{i c}^{3}-R_{i c-1}^{3}\right)\right] .
\end{aligned}
$$

The mean density of the inner core reads as

$$
\bar{\rho}_{s}=\frac{m_{s}}{\operatorname{Vol} D_{s}}=\frac{\rho_{1} R_{1}^{3}+\ldots+\rho_{i c}\left(R_{i c}^{3}-R_{i c-1}^{3}\right)}{R_{i c}^{3}} .
$$

The amplitude ratio of the mantle to the inner core (Eq. (9)) can be written as

$\eta=\frac{m_{s}-\rho_{f} \operatorname{Vol} D_{s}}{\left(m_{m}+m_{f}\right)+\rho_{f} \operatorname{Vol} D_{s}}$.

From Eqs. (B.1) and (B.2), with $\rho_{f}=\rho_{i c+1}$, we obtain

$$
\eta=\frac{\left(\rho_{1}-\rho_{2}\right) R_{1}^{3}+\ldots+\left(\rho_{i c}-\rho_{i c+1}\right) R_{i c}^{3}}{\left(\rho_{i c+1}-\rho_{i c+2}\right) R_{i c+1}^{3}+\ldots+\rho_{N} R_{N}^{3}}=\frac{\sum_{j=1}^{i c}[\rho]_{j} R_{j}^{3}}{\sum_{j=i c+1}^{N}[\rho]_{j} R_{j}^{3}},
$$

where $[\rho]_{j}=\rho_{j}-\rho_{j+1}, j=1, \ldots, N-1,[\rho]_{N}=\rho_{N}$.

Equation (B.4) is exactly the same formula as derived in Coyette et al. (2012, Eq. (3)).

\footnotetext{
${ }^{12}$ In the main part of the text we have denoted $R_{i c}, R_{i c+1}, R_{N}$, and $\rho_{i c+1}$ as $d_{s}, d_{f}, d_{m}$, and $\rho_{f}$, respectively.
} 